\title{
Dynamics of trapped two-component Fermi gas : temperature dependence of the transition from the collisionless to the collisional regime
}

Citation for published version (APA):

Toschi, F., Vignolo, P., Succi, S., \& Tosi, M. P. (2003). Dynamics of trapped two-component Fermi gas : temperature dependence of the transition from the collisionless to the collisional regime. Physical Review $A$ : Atomic, Molecular and Optical Physics, 67(4), 041605-1/4. https://doi.org/10.1103/PhysRevA.67.041605

DOI:

10.1103/PhysRevA.67.041605

Document status and date:

Published: 01/01/2003

Document Version:

Publisher's PDF, also known as Version of Record (includes final page, issue and volume numbers)

\section{Please check the document version of this publication:}

- A submitted manuscript is the version of the article upon submission and before peer-review. There can be important differences between the submitted version and the official published version of record. People interested in the research are advised to contact the author for the final version of the publication, or visit the DOI to the publisher's website.

- The final author version and the galley proof are versions of the publication after peer review.

- The final published version features the final layout of the paper including the volume, issue and page numbers.

Link to publication

\footnotetext{
General rights

- You may freely distribute the URL identifying the publication in the public portal. follow below link for the End User Agreement:

www.tue.nl/taverne

\section{Take down policy}

If you believe that this document breaches copyright please contact us at:

openaccess@tue.nl

providing details and we will investigate your claim.
}

Copyright and moral rights for the publications made accessible in the public portal are retained by the authors and/or other copyright owners and it is a condition of accessing publications that users recognise and abide by the legal requirements associated with these rights.

- Users may download and print one copy of any publication from the public portal for the purpose of private study or research.

- You may not further distribute the material or use it for any profit-making activity or commercial gain

If the publication is distributed under the terms of Article 25fa of the Dutch Copyright Act, indicated by the "Taverne" license above, please 


\title{
Dynamics of trapped two-component Fermi gas: Temperature dependence of the transition from collisionless to collisional regime
}

\author{
F. Toschi ${ }^{1,2}$ P. Vignolo,${ }^{3}$ S. Succi, ${ }^{1,3}$ and M. P. Tosi ${ }^{3}$ \\ ${ }^{1}$ Istituto per le Applicazioni del Calcolo, CNR, Viale del Policlinico 137, I-00161 Roma, Italy \\ ${ }^{2}$ INFM, Sezione di Roma “Tor Vergata," Via della Ricerca Scientifica 1, I-00133 Roma, Italy \\ ${ }^{3}$ NEST-INFM and Classe di Scienze, Scuola Normale Superiore, Piazza dei Cavalieri 7, I-56126 Pisa, Italy
}

(Received 25 October 2002; published 25 April 2003)

\begin{abstract}
We develop a numerical method to study the dynamics of a two-component atomic Fermi gas trapped inside a harmonic potential at temperature $T$ well below the Fermi temperature $T_{F}$. We examine the transition from the collisionless to the collisional regime down to $T=0.2 T_{F}$ and find a good qualitative agreement with the experiments of B. DeMarco and D.S. Jin [Phys. Rev. Lett. 88, 040405 (2002)]. We demonstrate a twofold role of temperature on the collision rate and on the efficiency of collisions. In particular, we observe a hitherto unreported effect, namely, the transition to hydrodynamic behavior is shifted towards lower collision rates as temperature decreases.
\end{abstract}

DOI: 10.1103/PhysRevA.67.041605

PACS number(s): 05.30.Fk, 71.10.-w

Recent experiments at JILA [1] on the collisions between two oscillating spin-polarized components of a Fermi gas of

${ }^{40} \mathrm{~K}$ atoms have shown that this setup is an important tool for investigating the dynamics of dilute quantum gases. These experiments have given evidence for a transition from collisionless (zero-sound) to hydrodynamical (first-sound) behavior: the measured damping time $\tau$ becomes very long at both low and large values of the estimated collision rate. The transition from the hydrodynamic to an intermediate regime has also been followed with decreasing temperature and the effect of Pauli blocking of the collisions from increasing occupation of final states has been exhibited at low temperature.

Various numerical experiments have addressed the dynamics of a thermal cloud of bosons [2], even in the presence of a Bose-Einstein condensate [3] or of cold spin-polarized fermions interacting with a condensate via mean field [4]. Fermion molecular dynamics (FMD) has been developed [5] as a quasiclassical model for treating problems such as ionatom collisions or formation of antiprotonic atoms. However, FMD is not adequate to deal with dilute fermionic systems, such as the neutral atomic gas under harmonic confinement realized in Ref. [1], and an approach explicitly acknowledging the diluteness and other characteristics of such a system is needed. Some work along these lines was developed in Ref. [6] using the semiclassical Boltzmann equations as a function only of the energy, to describe the evaporative cooling process. However, this simplified approach allows only a very limited description of the dynamics of the fermionic particles.

To the best of our knowledge, the present work reports the first numerical study directed to the transport properties of ultracold Fermi gases.

In this paper, we solve the Vlasov-Landau equations (VLE) for two-component fermionic Wigner distributions. As in FMD [5] or in numerical studies of the dynamics of thermal bosons [2-4], the quantum-mechanical fluid is treated by a particle-dynamics approach. We proceed along the path traced for a single spin-polarized Fermi gas [4], by duplicating it and introducing mean-field interactions and collisions between the two components. A major highlight of our numerical method is the strategy used to deal with collisional events. We develop a locally adaptive importancesampling technique, which allows us to handle collisional interactions faster than in standard Monte Carlo techniques by several orders of magnitude.

Owing to this computational advance, we are able to examine collisional effects down to $T / T_{F} \sim 0.2$, in a region of temperature $T$ well below the Fermi temperature $T_{F}$ where Pauli blocking would normally grind the simulation to a halt because of numerical attrition problems (basically through a saturation of phase space resulting in vanishing efficiency of the Monte Carlo sampling). Moreover, since we focus on the JILA setup [1] where the axial symmetry is maintained during the experiment, we can use an axially symmetric code with two-dimensional collisions, in which the angular degree of freedom is taken into account via an effective weight. The resulting code permits us to study the collisional properties of the two-component Fermi gas as functions of both temperature $T$ and quantum collision rate $\Gamma_{q}$. The numerical approach has full control over additional variables that are experimentally unaccessible. In particular, while in the experiments the classical collision rate can easily be estimated but the fully quantal collision rate $\Gamma_{q}$ remains unknown, in the numerical approach both classical and quantum collision rates can be counted step by step. Because of this control of the collision rates we are able to observe that, even if most collisions become forbidden classically and by the Pauli principle as temperature is lowered, the few collisions that can occur involve particles increasingly clustered around the Fermi level. The result is a kind of "catalytic effect" in which these few collisions suffice to drive the gas from the collisionless to the hydrodynamic regime.

The model and its solution. Here we write the VLE for two interacting Fermi gases and describe the algorithm used in their numerical solution. We summarize some points that have been described in more detail in Ref. [4] and point out how the difficulties raised by the Fermi statistics are handled in our method.

The two fermionic components in external potentials $V_{e x t}^{(j)}(\mathbf{r})$ are described by the distribution functions $f^{(j)}(\mathbf{r}, \mathbf{p}, t)$ with $j=1$ or 2 . These obey the kinetic equations 


$$
\partial_{t} f^{(j)}+\frac{\mathbf{p}}{m} \cdot \nabla_{\mathbf{r}} f^{(j)}-\nabla_{\mathbf{r}} U^{(j)} \cdot \nabla_{\mathbf{p}} f^{(j)}=C_{12}\left[f^{(j)}\right],
$$

where the mean-field Hartree-Fock (HF) effective potential is $U^{(j)}(\mathbf{r}, t) \equiv V_{e x t}^{(j)}(\mathbf{r})+g n^{(\bar{j})}(\mathbf{r}, t)$ with $\bar{j}$ denoting the species different from $j$. Here we have set $\hbar=1$ and $g=2 \pi a / m_{r}$, with $a$ being the $s$-wave scattering length between two atoms and $m_{r}$ is the reduced mass, and $n^{(j)}(\mathbf{r}, t)$ is the spatial density given by integration of $f^{(j)}(\mathbf{r}, \mathbf{p}, t)$ over momentum.

Collisions between atoms of the same spin can be neglected at low temperature, so that in Eq. (1) the term $C_{12}$ involves only collisions between particles with different polarizations:

$$
\begin{aligned}
C_{12}\left[f^{(j)}\right] \equiv & 2(2 \pi)^{4} g^{2} / V^{3} \sum_{\mathbf{p}_{2}, \mathbf{p}_{3}, \mathbf{p}_{4}} \Delta_{\mathbf{p}} \Delta_{\varepsilon}\left[\bar{f}^{(j)} \bar{f}_{2}^{(\bar{j})} f_{3}^{(j)} f_{4}^{(\bar{j})}\right. \\
& \left.-f^{(j)} f_{2}^{(\bar{j})} \bar{f}_{3}^{(j)} \bar{f}_{4}^{(\bar{j})}\right]
\end{aligned}
$$

with $f^{(j)} \equiv f^{(j)}(\mathbf{r}, \mathbf{p}, t), \bar{f}^{(j)} \equiv 1-f^{(j)}, f_{i}^{(j)} \equiv f^{(j)}\left(\mathbf{r}, \mathbf{p}_{i}, t\right)$, and $\bar{f}_{i}^{(j)} \equiv 1-f_{i}^{(j)}$. Here, $V$ is the volume occupied by the gas and the factors $\Delta_{\mathbf{p}}$ and $\Delta_{\varepsilon}$ are the usual $\delta$ functions accounting for the conservation of momentum and energy, with the energies given by $p_{i}^{2} / 2 m_{j}+U^{(j)}(\mathbf{r}, t)$.

The numerical procedure by which the VLE is advanced in time consists of three basic steps: (i) initialization of the fermionic distributions, (ii) propagation in phase space, and (iii) collisional interactions.

The initial distributions in equilibrium at the bottom of a harmonic trap are generated by using the HF expression

$$
f_{e q}^{(j)}(\mathbf{r}, \mathbf{p})=\left\{\exp \left[\beta\left(\frac{p^{2}}{2 m_{j}}+U^{(j)}(\mathbf{r})-\mu^{(j)}\right)\right]+1\right\}^{-1},
$$

where $\beta=1 / k_{B} T$ and $\mu^{(j)}$ is the chemical potential of species $j$ [7]. The particle densities entering $U^{(j)}(\mathbf{r})$ are to be determined self-consistently by integration over momenta, and the momentum distributions of the two clouds need generating.

To exploit the axial symmetry of the system, we first define the angularly integrated particle densities $\mathcal{N}^{(j)}(r, z)$ $=2 \pi r n^{(j)}(r, z)$ on a $\{r, z\}$ grid in cylindrical coordinates and we move on to a particles-in-cell description by locating a number $\mathcal{N}^{(j)}(r, z) \Delta r \Delta z$ of fermions in each cell of volume $\Delta r \Delta z$ [4]. A momentum distribution with low statistical noise is generated by representing each fermion by means of $N_{q}$ computational particles ("quarks"). The $i$ th quark is located at point $\left\{p_{i r}, p_{i \theta}, p_{i z}\right\}$ in momentum space by using Monte Carlo sampling and making sure that each cell of volume $h^{3}$ is occupied by no more than $N_{q}$ quarks. This control in three-dimensional (3D) phase space is transferred to $2 \mathrm{D}$ by imposing a maximum number $w N_{q}$ of quarks in the 2D cell $\left\{\Delta r, \Delta z, \Delta p_{r}, \Delta p_{z}\right\}$ of volume $h^{2}$. Here the weight $w=\operatorname{int}\left(2 p_{F} r / \hbar\right)$ takes into account that the number of available cells in 2D depends on the radial position and on the number of particles through the Fermi momentum $p_{F}$ $=\sqrt{2 m \hbar k_{B} T_{F}}$.

In the propagation step, the two clouds are rigidly displaced from the center of the trap along the $z$ direction and start evolving in time in the $\{r, z\}$ plane by performing oscillations at their respective frequencies. We exploit the fact that by symmetry the average value of $p_{\theta}$ for each value of $r$ is zero and does not change in time, to decouple the variable $p_{\theta}$ from the equations of motion. We make the approximation that the angular momentum of each quark is left unchanged by the collisional events and take into account the third dimension in the exclusion principle by suppressing collisions whose final states are occupied by more than $w N_{q}$ quarks. At each time step, the quarks are moved by the confinement and the mean-field forces by using a Verlet algorithm on a grid of mesh spacing $d x>v d t$, where $v$ is a typical particle velocity and $d t$ is the time step. This inequality is dictated by the accuracy and the stability of the propagation step [4]. No exclusion constraint is applied during this step: this does not lead to any appreciable deviation from the Fermi statistics down to $0.2 T_{F}$, where the system is still sufficiently dilute. This has been checked by monitoring violations of the Pauli principle at each time step.

Finally, we come to the collision step, which involves most of the innovative aspects of our scheme. Collisions are tracked on a grid of mesh spacing $\delta x$ of the order of the de Broglie wavelength $\lambda_{B}$, which is smaller than the particle mean free path $l$ and larger than the spacing $d x$ of the propagation mesh $\left(l>\delta x \sim \lambda_{B}>d x\right)$. The first inequality enhances the statistical accuracy of the collision step, whereas coarse graining with respect to the propagation step avoids the need for the Pauli-principle constraints, at least down to $0.2 T_{F}$.

Before turning to the results, we add some computational details on how a speedup of the code by several orders of magnitude has been achieved. The number of probable collisions between all possible pairs of quarks belonging to the two species in each cell of volume $h^{2}$ is evaluated at each computational time step as $d N_{\text {coll }}=d t \sqrt{N_{1} N_{2}} \Sigma_{i j} v_{i j} \sigma_{i j}$. Here $v_{i j}$ is the magnitude of the relative speed of particle $i$ of species 1 and particle $j$ of species $2, \sigma_{i j}$ is the corresponding differential cross section, and $N_{1}\left(N_{2}\right)$ is the number of particles of species 1 (2) in the given spatial cell. If $d N_{\text {coll }}$ $<1$, the collision probability is accumulated over the subsequent time steps until an integer number $d I_{\text {coll }}$ $\equiv \operatorname{int}\left(d N_{\text {coll }}\right)$ of collisions occurs. Within each spatial cell the pairs of particles are ordered according to the value of their classical collision probability, from largest to smallest. The acceptance rate of the Monte Carlo sampling is enhanced by two to three orders of magnitude at each step by filtering out pairs with classical probability smaller than a given threshold. This is a form of importance sampling and the threshold is dynamically adjusted cell-by-cell in such a way as to guarantee the correct supply of $d I_{\text {coll }}$ collisions at each time step. The collision probability becomes smaller than the classical one after multiplication by the quantum suppression factor $1-N\left(r, z, p_{r}, p_{z}\right) /\left(w N_{q}\right)$ due to the occupancy of the final state, and consequently the selection of the most likely pairs to collide becomes essential. To this purpose, each particle is allowed to collide only with the partner that maximizes the product $v_{i j} \sigma_{i j}$. The original pool of $N_{1} N_{2}$ collisions is cut down to $N_{1}$ collisions only, with a resulting additional speedup of at least one order of magnitude. 


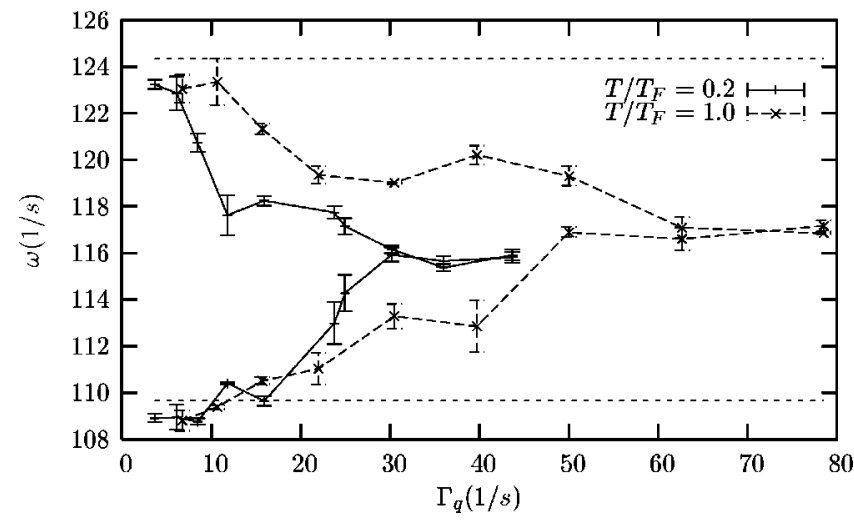

(a)

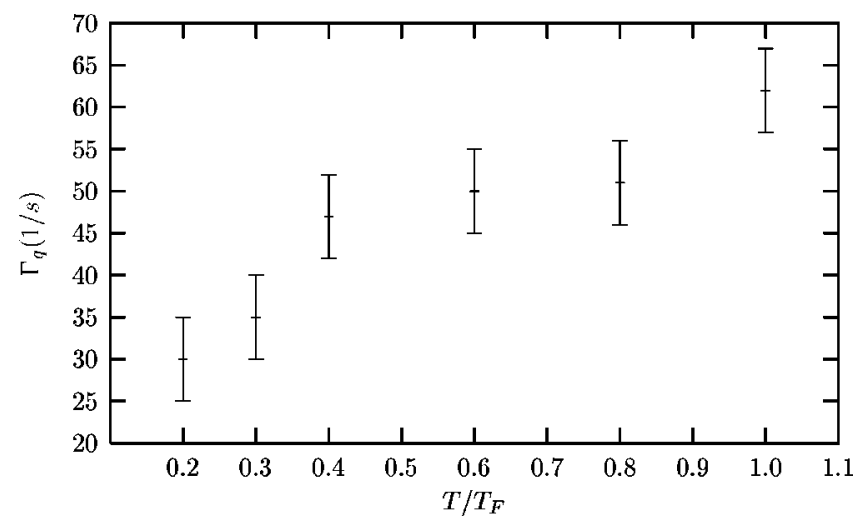

(b)

FIG. 1. (a) The oscillation frequencies $\omega$ (in units of $s^{-1}$ ) as functions of the quantum collision rate $\Gamma_{q}$ (in units of $\mathrm{s}^{-1}$ ) for the two components of the gas at $T=T_{F}(\times)$ and $T=0.2 T_{F}(+)$. The horizontal dashed lines show the bare trap frequencies. (b) The collision rate $\Gamma_{q}$ at frequency locking as a function of temperature $T$ (in units of $T_{F}$ ).

Results. As a first application of the numerical method, we have considered 200 magnetically trapped ${ }^{40} \mathrm{~K}$ atoms, which are represented by a total number of $4 \times 10^{3}$ quarks. As in the JILA experiments [1], the atoms are equally shared among two different spin states $\left(m_{f}=9 / 2\right.$ and $\left.m_{f}=7 / 2\right)$ in harmonic traps with slightly different longitudinal frequencies $\left(\omega_{9 / 2}\right.$ $=2 \pi \times 19.8 \mathrm{~s}^{-1}$ and $\left.\omega_{7 / 2}=2 \pi \times 17.46 \mathrm{~s}^{-1}\right)$. When the two clouds after initialization are rigidly displaced from the center of the traps, in the absence of interactions they would keep oscillating at their respective trap frequencies without damping.

The collision rate $\Gamma_{q}$ is independently varied by changing the scattering length $a$, thus mimicking the exploitation of a suitable Feshbach resonance [8]. To offset the difference between the number of atoms used in the simulation $(N$ $=200$ ) and that in the experiments $\left(N_{\text {exp }} \sim 10^{6}\right)$, the reference value of the off-resonant scattering length is scaled by a factor $\left(N_{\text {exp }} / N\right)^{1 / 2} \sim 10^{2}$, thus producing a system with essentially the same collision rate and mean-field potential as in Ref [1]. The transition from the collisionless to the collisional regime, as driven by varying the scattering length at various temperatures, is shown both in the plot of the frequency of the dipole mode for the two components in Fig.

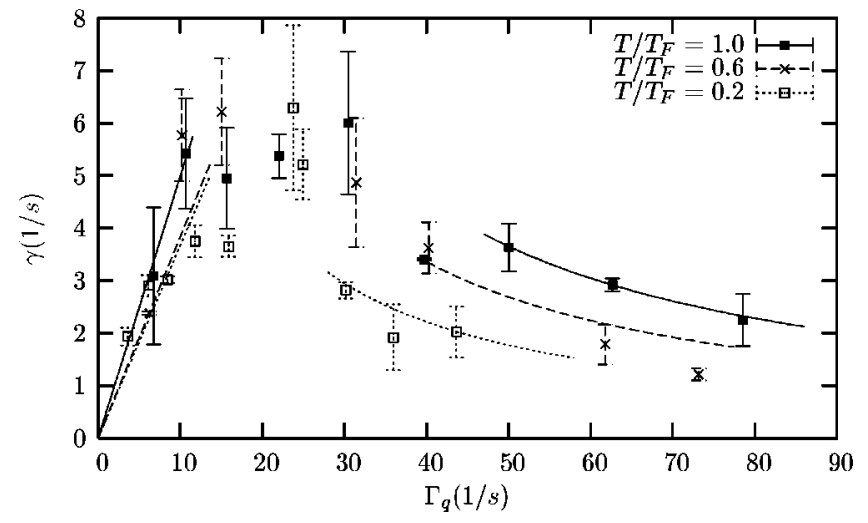

FIG. 2. The damping coefficient $\gamma$ (in units of $\mathrm{s}^{-1}$ ) as a function of the collision rate $\Gamma_{q}$ (in units of $\mathrm{s}^{-1}$ ) for $T=T_{F}$ (filled squares), $T=0.6 T_{F}$ (crosses), and $T=0.2 T_{F}$ (empty squares). In the collisionless region, $\gamma$ has been fitted by the function $\gamma\left(\Gamma_{q}\right)=\alpha_{1} \Gamma_{q}$ and in the collisional regime by the function $\gamma\left(\Gamma_{q}\right)=\alpha_{2} / \Gamma_{q}$. The fits are shown by a continuous line for $T=T_{F}$, a dashed line for $T$ $=0.6 T_{F}$, and a dotted line for $T=0.2 T_{F}$.

1(a) and in the plot of the damping rate $\gamma=1 / \tau$ of the axial motion of the centers of mass $z_{\mathrm{cm}}^{(j)}(t)$ in Fig. 2 .

In Fig. 1(a), the oscillation frequencies $\omega_{j}$ at $T=T_{F}$ and $T=0.2 T_{F}$, with $k_{B} T_{F}=\hbar \omega_{9 / 2}\left(6 N_{9 / 2}\right)^{1 / 3}$, are evaluated by fitting $z_{\mathrm{cm}}^{(j)}(t)$ with the functions $z_{0} \cos \left(\omega_{j} t\right) e^{-\gamma t}$. At very low $\Gamma_{q}$ the dipole mode frequencies are given by the corresponding trap frequencies with a shift due to the mean-field potential. At intermediate values of $\Gamma_{q}$, the data points show large fluctuations, due to the fact that in this region just a few collisions can drastically alter the motion of the clouds. However, the trend towards a locking of the two dipole mode frequencies at large $\Gamma_{q}$ is very clear and the location of the locking is identified with reasonable accuracy.

The main physical result of this study is the shift of the locking transition to lower $\Gamma_{q}$ as temperature is decreased. This is shown in Fig. 1(b). This effect is related to the Fermi statistics: at low temperature, the collisions involve particles on a narrower region around the Fermi level and have a greater impact on the global dynamics of the gas. A smaller number of collisions is needed to produce locking of the two interacting species. Here we wish to emphasize that we were able to gain insight into this unforeseen effect, thanks to our detailed numerical methods, which allowed us to precisely measure the full quantum collision rate at each time step.

The damping rate $\gamma$ obtained from the fit of $z_{\mathrm{cm}}^{(j)}(t)$ is essentially the same for the two components and is shown in Fig. 2. We have also evaluated the correlation function $\phi(t)=\left\langle\left|\mathcal{Z}_{\mathrm{cm}}^{(j)}\left(t^{\prime}\right)\right|\left|\mathcal{Z}_{\mathrm{cm}}^{(j)}\left(t^{\prime}+t\right)\right|\right\rangle$ between the magnitudes of the turning points $\mathcal{Z}_{\mathrm{cm}}^{(j)}$, which decays exponentially as exp $(-\gamma t)$ at large $t$. This estimate of $\gamma$ is in good agreement with that obtained from the center-of-mass motions, but yields a less noisy signal in the intermediate region. In the collisionless regime the damping rate increases linearly with $\Gamma_{q}$, while in the collisional one it scales like $\Gamma_{q}^{-1}$. This is seen in Fig. 2, which also shows again that the hydrodynamic regime is reached at lower $\Gamma_{q}$ as $T$ is lowered.

The collision rate $\Gamma_{q}$ can be driven by cooling at fixed 


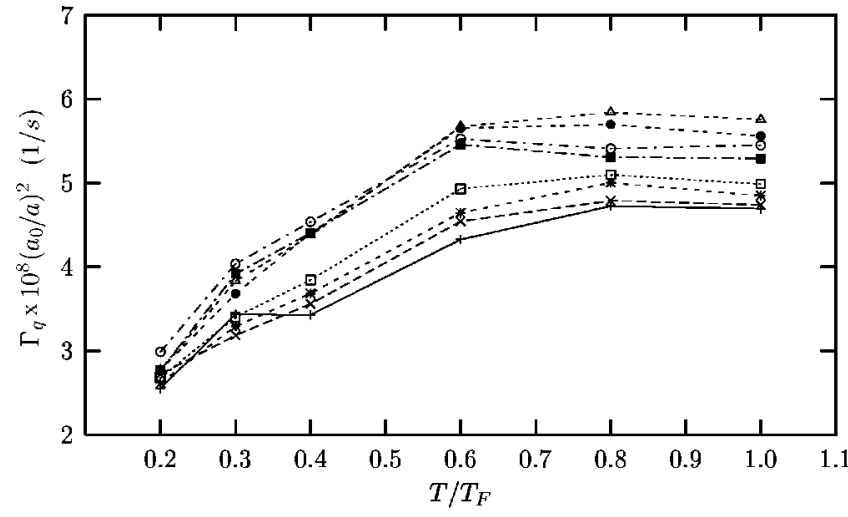

FIG. 3. The collision rate $\Gamma_{q}$ (in units of $\mathrm{s}^{-1}$ ) scaled by the factor $10^{8}\left(a_{0} / a\right)^{2}$ with $a_{0}$ being the Bohr radius, as a function of temperature $T$ (in units of $T_{F}$ ) for various values of the scattering length $a$. From bottom to top: $a=(12,15,18,21,24,27,30,33)$ $\times 10^{3} a_{0}$.

scattering length, as is seen from Fig. 3. The various curves, after scaling by a factor proportional to the classical cross section, show a residual weak dependence on $a$, which is due to the mean-field interaction between the two overlapping fermionic components. The trend of these curves is mostly classical and only at very low temperature $(T$ $=0.3 T_{F}-0.2 T_{F}$ ) Pauli blocking becomes manifest, as signalled by the collapse of the curves into a single one. Our results thus suggest new experiments, in which the collision rate is changed via a thermal drive.

The implication of the results shown in Figs. 2 and 3 is that this thermal drive rests upon the increasing importance of a decreasing set of "strategic" collisions involving particles in states clustered around the Fermi level. Our data also indicate that thermal cooling will need to reach temperatures below $0.2 T_{F}$ in order to see the complete transition from the collisional to the collisionless regime.

Efforts to develop a concurrent code, including the Pauli principle in the Lagrangian evolution to treat the collisional Fermi gas well below $0.2 T_{F}$, are currently under way. A completely three-dimensional approach will allow further studies, including the investigation of anisotropic fluctuations. The extension of the code to the case of negative scattering lengths will also allow us to make contact with current experiments on the anisotropic expansion of a cigar-shaped gas in the proximity of a Feshbach resonance [9].

We thank Dr. A. Minguzzi for helpful discussions and acknowledge support from INFM through the PRA2001 Program.
[1] S.D. Gensemer and D.S. Jin, Phys. Rev. Lett. 87, 173201 (2001); B. DeMarco and D.S. Jin, ibid. 88, 040405 (2002).

[2] H. Wu and C.J. Foot, J. Phys. B 29, L321 (1996).

[3] T. Nikuni, E. Zaremba, and A. Griffin, Phys. Rev. Lett. 83, 10 (1999).

[4] P. Vignolo, M.L. Chiofalo, S. Succi, and M.P. Tosi, J. Comput. Phys. 182, 368 (2002).

[5] L. Wilets and J.S. Cohen, J. Contemp. Phys. 39, 163 (1998); H. Feldmeier and J. Schnack, Rev. Mod. Phys. 72, 655 (2000).
[6] W. Geist and T.A.B. Kennedy, Phys. Rev. A 65, 063617 (2002).

[7] M. Amoruso, I. Meccoli, A. Minguzzi, and M.P. Tosi, Eur. Phys. J. D 7, 441 (1999).

[8] T. Loftus, C.A. Regal, C. Ticknor, J.L. Bohn, and D.S. Jin, Phys. Rev. Lett. 88, 173201 (2002).

[9] K.M. O'Hara, S.L. Hemmer, M.E. Gehm, S.R. Granade, and J.E. Thomas, Science 298, 2179 (2002); C.A. Regal and D.S. Jin, e-print cond-mat/0302246. 